\title{
The DELTA photon counting camera concept
}

\author{
S. Morel and L. Koechlin \\ Laboratoire d'Astrophysique de Toulouse, Observatoire Midi-Pyrénées, 14 avenue Édouard Belin, F-31400 Toulouse, France \\ e-mail: morel@obs-mip.fr, koechlin@obs-mip.fr
}

Received June 18; accepted December 17, 1997

\begin{abstract}
In this paper we present new ideas for a high sensitivity array detector, which yields the space and time coordinates of photo-events at sustained count rates superior to one million per second. The DELTA camera (Detector Enhancement by Linear-projections on Three Axes) has been initially designed for astronomy. However, it has advantages for a wide range of high-resolution problems in the visible and near I.R. It is noticeable for its optical design, its smooth flat field and its very high temporal resolution and throughput in photon counting mode. The resolution of the prototype described is $512 \times 591$ pixels in space and $2.6 \mu \mathrm{s}$ in time. The principle of this detector is based on a projection - back-projection scheme (the $\Delta$ process) and on the use of three one-dimensional CCDs. We describe the technical solutions which could lead to an operational prototype. From numerical simulations of the $\Delta$-process, we give some expected characteristics of this camera.
\end{abstract}

Key words: instrumentation: detectors - techniques: interferometric

\section{Introduction}

Although a majority of cosmic phenomena is slow enough to allow imaging with long exposures, ground-based highresolution techniques require very short frame times, to deal with atmospheric seeing and reach the theoretical limit in resolution for large telescope diameters (Roggemann et al. 1997). Stellar speckle interferometry techniques (Labeyrie 1970; Knox \& Thompson 1974; Lohmann et al. 1983), use large series of images, each one with a $10 \mathrm{~ms}$ average integration time. Adaptive optics wave-front sensors (Hardy 1978; Roddier 1988) require even shorter frame times. Fringe tracking in optical multiple aperture interferometers (Koechlin et al. 1996) also

Send offprint requests to: S. Morel require high spatial and temporal resolution. A new application, requiring high data rate photon counting capabilities, is the "dark speckle" technique for exo-planet detection (Labeyrie 1995). This single aperture method consists in doing statistics on photons collected in each pixel of the field. It involves a photon flux at the performance limit of existing cameras, as noted from recent experiments (Boccaletti \& Labeyrie 1997).

At short integration times, quantum limits are often reached, and photon counting detectors with microchannel plates (MCP) must be employed. Each frame is then represented by a cluster of detected photo-events. Historically, the first photon detector dedicated to speckle interferometry was an intensified film camera, built by Gezari (1972). Later, video systems were used (Blazit et al. 1977), yielding clipped frame signals where photon positions were marked by a logical 1 . Later, detectors were designed, yielding the coordinates of photo-events in the image plane, thus allowing compact data storage and online processing. Surprisingly, the earliest device providing such data was dedicated to a long exposure task: spectroscopy (Boksenberg et al. 1972).

Among the cameras currently used for both speckle and multiple aperture interferometry, providing direct photon coordinates, are the CP40 (Blazit 1986), the Resistive Anode Camera, or "Ranicon" (Clampin et al. 1988), the PAPA camera (Papaliolios \& Mertz 1982), the MAMA camera (Timothy \& Bybee 1975), the "Wedgeand-Strip" camera (Martin et al. 1983), and the delay line camera (Lampton et al. 1987). The main problem of the CP40 is its limited maximum output rate of photon coordinates $(25000 \mathrm{ph} / \mathrm{s}$ - photons per second - $)$. This limitation, which is due to the photon coordinate determination process causes artifacts in image autocorrelations. The Ranicon is even slower, limited to $10000 \mathrm{ph} / \mathrm{s}$. Beyond this limit, pulse pile-up on the anode causes incorrect photon coordinate measurements. With these cameras the maximal signal-to-noise ratio is often reached on bright objects or with large apertures instruments, such as GI2T (Mourard et al. 1994). Although the PAPA 
camera can work at higher photon rate, it has field uniformity problems (due to the limited precision of the mask reimaging), as described by Lawson (1994). Wedge-andStrip cameras are also affected by pulse pile-up, and by external magnetic deflection, requiring careful shielding (Timothy 1983).

Besides the maximum photon rate, another relevant characteristic of a photon counting camera is the temporal resolution (i.e. photo-event dating accuracy). Recently developed tools dealing with spatiotemporal photon coordinates could improve fringe tracking, especially in the case of space-borne interferometers (Koechlin 1985; Vakili \& Koechlin 1989), or for moving object recognition (Morel $\&$ Koechlin 1997). These techniques require a higher temporal resolution than achievable by existing ICCD matrix photon counting cameras. Although MAMA camera prototypes (Timothy 1985) yield high maximum photon rate $\left(10^{6} \mathrm{ph} / \mathrm{s}\right)$, and high spatial and temporal resolutions (up to $4096 \times 4096$ pixels, with 100 ns event timing accuracy), their cost of realization makes this kind of equipment affordable for major projects only. Improvements of Wedge-and-Strip cameras have lead to hybrid detectors (Lampton et al. 1987) using a delay line approach for the $x$ determination, and the charge partition system of Wedgeand-Strip for the $y$ determination. The delay line system is very promising as it allows maximum count rates and temporal resolutions matching the MAMA performances. Nevertheless, an accurate delay measurement system is required, like the 4 ps resolution time-to-digit converter built by Lampton \& Raffanti (1994). With a double delay line (Raffanti \& Lampton 1993), the $y$-resolution of the camera is enhanced by reducing the anode capacitance. A detector using delay lines only for both $x$ and $y$ determination (crossed serpentine delay lines) has been recently built by Friedman et al. (1996).

Building the DELTA camera, our goal is therefore twofold. First, to provide high rates of accurate photon coordinates for speckle interferometry, dark speckle, fringe detection, and wave-front sensing. Second, to achieve high temporal resolution in order to test spatiotemporal methods (Morel \& Koechlin 1997) with the best possible accuracy (actually, in the case of the DELTA camera, these two aspects are tied, as we shall see). Our goal is constrained by the requirements of low-cost and reliable technology. We have therefore selected commercially available components, and chosen solutions to avoid using high-precision photo-etching or high-vacuum equipment.

\section{The geometric concept of the DELTA camera}

\subsection{Synchronous and asynchronous cameras}

Like many other photon counting devices, the DELTA camera will use an image intensifier providing a gain of about 1 million, producing detected photo-events as bright spots on a fast decay phosphor. The challenge is to translate these intensified photo-events into numerical coordinates as fast as possible, in order to achieve the highest data flow and temporal resolution.

Most of photon counting cameras, that we will refer to "asynchronous", like the Ranicon, PAPA, MAMA, or delay-line process only one photon at a time: if two or more intensified photo-events are simultaneously present in the field, the coordinate computation system fails, yielding no data or incorrect coordinates. Except for the MAMA and the delay-line cameras, the data flow is thus limited by: first, either the phosphor decay time $(0.5 \mu \mathrm{s})$ or the resistive anode decay $(50 \mu \mathrm{s})$, and second, the photon coordinate determination process ( 1 to $10 \mu \mathrm{s})$.

Other cameras, "synchronous", like the CP40 use a 2-dimensional ICCD and can process many photons in a single frame. They suffer from a trade-off between spatial resolution and read time of the CCD array: typically 5 to $20 \mathrm{~ms}$. They also suffer from an artifact causing problems in second order moment imaging techniques. Due to the long frame time, there is a non-negligible probability that two detected photons fall close enough in a CCD frame (although not onto the same pixel) to be seen as a single photon, or no photon at all by the coordinate determination electronics.

\subsection{The Projection - Back-Projection scheme in the DELTA camera}

To solve these problems, the DELTA synchronous camera uses three fast linear CCD chips (each 1024 by 1 pixels and $2.6 \mu \mathrm{s}$ frame read time). It may detect and locate several photons simultaneously in each frame, with a 512 by 591 pixels hexagonal field.

The principle is as follows: an intensified frame containing $N$ photons detected between times $t$ and $t+\Delta t$ is described by:

$$
F_{t}(x, y)=\sum_{i=0}^{N-1} \delta\left(x-x_{i}, y-y_{i}\right),
$$

where $\delta$ represents a Dirac distribution. The photon coordinates to be extracted from the frame are the triplets $\left(x_{i}, y_{i}, t_{i}\right) . t_{i}$ is set to $t$ for all the photons in the frame. $x_{i}$ and $y_{i}$ are extracted by a Projection - Back-projection scheme.

\subsubsection{Projection}

Three images of the intensified field are formed. These "images" are reduced to lines (orthogonal projections of the field) by the optical setup described in Sect. 5.2, and each line is directed to a CCD chip. Let $p_{\theta, \mathbf{M}}(x, y)$ be the projection operator defined by:

$$
p_{\theta, \mathbf{M}}(x, y)=\left(x-x_{\mathrm{M}}\right) \cos \theta+\left(y-y_{\mathrm{M}}\right) \sin \theta .
$$


It converts the coordinates $(x, y)$ of a photon into its projection on an axis. The axis onto which the projection is made is defined by its direction $\theta$ and the coordinates of its origin $M=\left(x_{\mathrm{M}}, y_{\mathrm{M}}\right)$. The coordinates of a photon could be retrieved from two orthogonal projections. However, if there is more than one photon in the field, there is not a unique solution to the problem of recovering a photon coordinate list from the two projection lists $L_{x}, L_{y}$. The information which associates one element of $L_{x}$ with the corresponding element of $L_{y}$ is missing. Therefore, the photon rate would be limited to one per frame. The solution is to reconstruct a "link table" between the two projection lists by projecting onto a third axis. Let $A, B$, $C$ be three axes onto which the photons are projected. To each photon coordinate vector $\boldsymbol{X}=(x, y)$ will correspond a projection vector $\boldsymbol{Y}=(a, b, c)$ such that:

$$
\left\{\begin{array}{l}
a=p_{\alpha, \mathrm{A}}(\boldsymbol{X})=\left(x-x_{\mathrm{A}}\right) \cos \alpha+\left(y-y_{\mathrm{A}}\right) \sin \alpha \\
b=p_{\beta, \mathrm{B}}(\boldsymbol{X})=\left(x-x_{\mathrm{B}}\right) \cos \beta+\left(y-y_{\mathrm{B}}\right) \sin \beta \\
c=p_{\gamma, \mathrm{C}}(\boldsymbol{X})=\left(x-x_{\mathrm{C}}\right) \cos \gamma+\left(y-y_{\mathrm{C}}\right) \sin \gamma .
\end{array}\right.
$$

The projection can be seen as a Radon transform with only three directions. It is done optically with the setup described in Sect. 5.2. It is a virtually null-time operation.

\subsubsection{Back-projection}

The redundancy in the projection vectors allows the recovery in most cases, the coordinate list from the projection lists. Choosing the projections operators such that $\alpha=0 ; \beta=2 \pi / 3 ; \gamma=4 \pi / 3$, and $\mathrm{A}=\mathrm{B}=\mathrm{C}=(0,0)$, yields the relation:

$$
a+b+c=0 .
$$

Thus, among all the possible triplets obtained by picking one number in each of the three projection lists, only those having a null-sum will correspond to a photon. This is the basis of the coordinate determination process in the DELTA camera. It can be summarized by: "Project optically the image on the sides of an equilateral triangle, detect the one-dimensional projections, then back-project numerically using the null-sum test". The equilateral triangle gave its name to the camera.

\subsubsection{Field}

Let $K$ be the side of the equilateral triangle used for the projections ( $K$ also corresponds to the quantization dynamics: each $\Delta$-coordinate is an integer ranging from $-K / 2$ to $K / 2-1)$. The set of points $(x, y)$ within the range of the projection $p_{\theta, 0}$ onto a segment of length $K$ is:

$$
\Omega_{\theta}=\left\{(x, y) /-\frac{K}{2} \leq x \cos \theta+y \sin \theta<+\frac{K}{2}\right\} .
$$

The field of the detector is: $\Omega_{\alpha} \cap \Omega_{\beta} \cap \Omega_{\gamma}$. As shown in Fig. 1, this field is a hexagon.

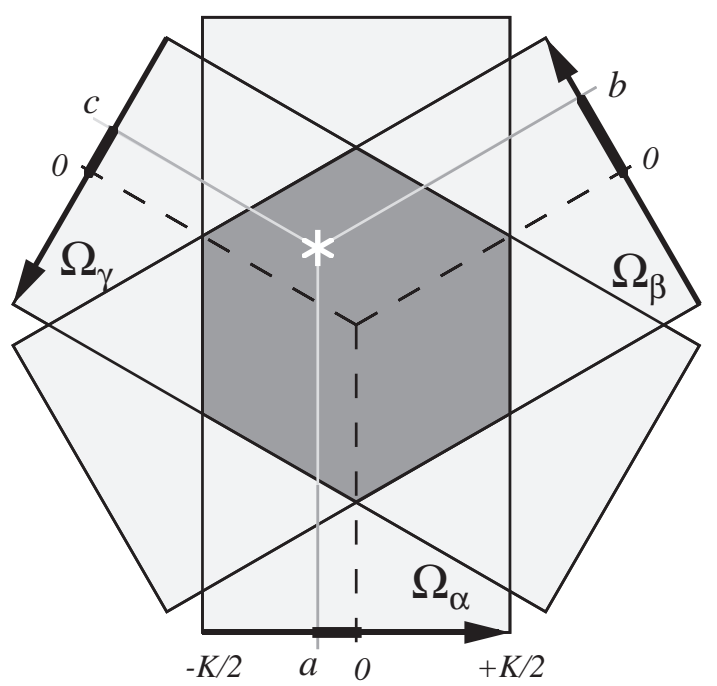

Fig. 1. Intersection of three stripes $\left(\Omega_{\alpha}, \Omega_{\beta}\right.$, and $\left.\Omega_{\gamma}\right)$ at $120^{\circ}$ angles forming the effective field of the DELTA camera (shaded hexagon). Projections (noted $a, b$, and $c$ ) of a point in the field (in white) onto the three corresponding axes

\section{Limitations of the null-sum test}

The data quantization on $\log _{2} K$ bits may cause $a+b+c \neq$ 0 for a given photo-event. Misdirected projections due to bad optical alignment, or small errors in the CCD spot ranging may have the same effect. A tolerance $\varepsilon$ must be set, replacing Eq. (4) by the double inequality:

$$
-\frac{\varepsilon}{2} \leq a+b+c \leq+\frac{\varepsilon}{2} \text {. }
$$

This may cause the null-sum test to fail and create incorrect photo-events. Suppose a photon having $\Delta$-projections $\left(a_{1}, b_{1}, c_{1}\right)$ which comply with the inequality:

$$
\left|a_{1}+b_{1}+c_{1}\right| \leq \varepsilon / 2 .
$$

There may exist lists $L_{\mathrm{A}}, L_{\mathrm{B}}$, and $L_{\mathrm{C}}$, such that changing one $\Delta$-coordinate among $a_{1}, b_{1}$, or $c_{1}$ by another one in the lists leaves Eq. (7) unchanged. The same thing may happen by changing two $\Delta$-coordinates (either $a_{1}$ and $b_{1}$, or $a_{1}$ and $c_{1}$, or $b_{1}$ and $c_{1}$ ). The back-projection process will therefore generate an extra photon. As this pseudo-photon is a crossover between two or three existing photons, we call it "cross-photon". There are two types of cross-photons. The first type regards those made by crossover of two existing photons (two $\Delta$-coordinates from the same existing photon). The second type is a crossphoton having its $\Delta$-coordinates originating from three different existing photons.

Type- 1 cross-photons are due to the non-zero tolerance $\varepsilon$. Type- 2 cross-photons occur with an increasing probability when three or more photons are present in the same frame.

In order to assess the frequency of occurence of crossphotons, one of us (SM) wrote a simulation software generating projections from random numerical "photons". 

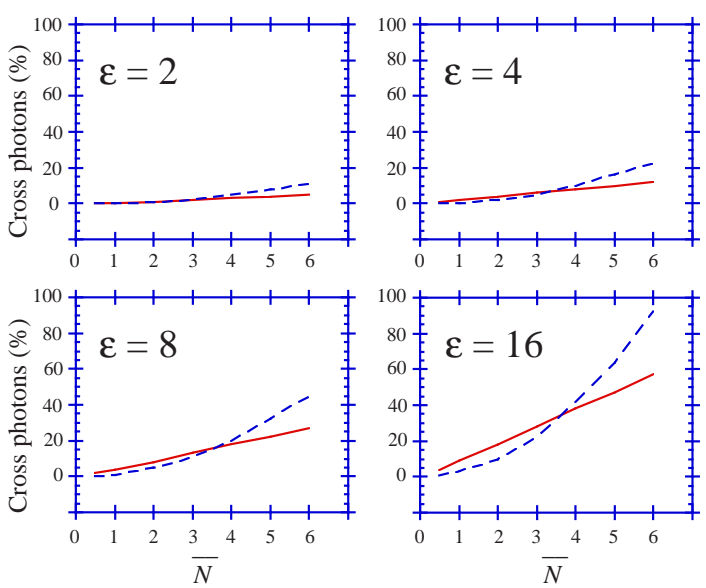

Fig. 2. Results of numerical simulations with a "Poisson" photon generator. Percentage (normalization by the number of incoming photo-events) of type-1 (solid line) and type-2 (dashed line) cross-photons generated, for different average numbers of photons per frame: $\bar{N}$, and null-sum test tolerance in pixels: $\varepsilon$

The number of generated photons per frame is variable and complies with Poisson's law. Given $\bar{N}$, the average number of photons per frame, this software draws a variable number $N$ of photons for each frame, such as:

$$
\operatorname{prob}(N=k)=\frac{\bar{N}^{k}}{k !} \exp (-\bar{N}) .
$$

We considered $\bar{N} \leq 5$ and we set $K=1024$, as in the prototype to be built (see Sect. 5). We measured the quantity of type- 1 and type- 2 cross-photons generated, normalized by the number of input photons. Figure 2 plots the results for several values of $\bar{N}$ and $\varepsilon$. It clearly appears that type- 2 cross-photons dominate for large $\bar{N}$.

\section{4. "Cross-cleaning"}

There is a solution for taking out cross-photons. It consists in selecting among the null-sum triplets only those having at least one $\Delta$-coordinate not shared with any other nullsum triplet. As each $\Delta$-coordinate of a cross-photon is shared, by definition, with a real photon, all cross-photons will be removed. The draw-back is that in some cases, valid photons may be removed.

Let $\left(a_{1}, b_{1}, c_{1}\right)$ the $\Delta$-coordinates of a real photon. If the frame contains a large number of photons, there may be three cross-photons with $\Delta$-coordinates: $\left(a_{1}, \ldots, \ldots\right)$, $\left(\ldots, b_{1}, \ldots\right)$, and $\left(\ldots, \ldots, c_{1}\right)$. Hence, this photon would be removed, causing a loss in overall quantum efficiency. With the simulator, we measured this attenuation factor after cross-cleaning. Measurements were made for different values of $\bar{N}$ and $\varepsilon$ (Fig. 3a). Poisson's law was used to draw the value of $N$ for each frame. To take into account a non-uniformly illuminated field, more subject to a quantum efficiency loss by cross-cleaning than a flat-field, we also used input photons from stellar speckle data acquired
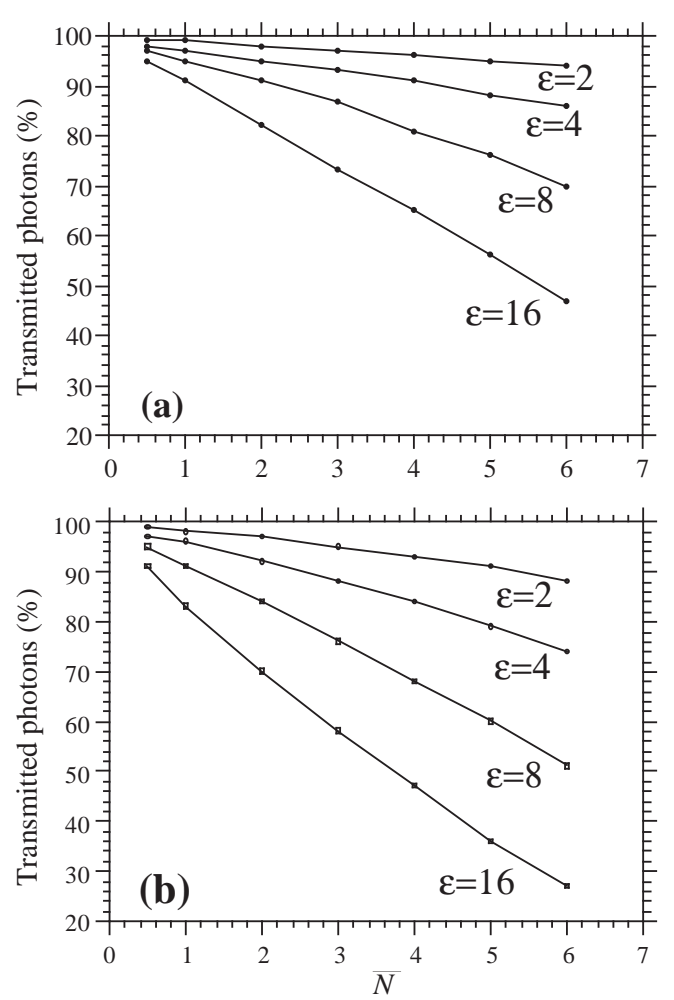

Fig. 3. Cross-cleaning removes the cross-photons but reduces the quantum efficiency. This figure shows the percentage of transmitted photons vs. mean number of photons per frame $(\bar{N})$ for different tolerances $\varepsilon$ and in case of: a) flat-field imaging, b) speckle imaging (unresolved star, $2^{\prime \prime}$ speckle pattern and $4^{\prime \prime}$ field)

with a Ranicon camera at a 2 meter diameter telescope (the V-Cygni star observation). The field illuminated during speckle observations is illustrated by Fig. 4. Poisson's law was used to draw photons sequentially from a speckle interferometry data file. Figure $3 \mathrm{~b}$ shows that a reduced field of illumination does not strongly affect the quantum efficiency: it remains fair at $\varepsilon \leq 4$ and $\bar{N} \leq 5$.

Cross-photons are not noticeable in the integrated images, but they cause an artifact in the integrated autocorrelations. This would cause problems for second order moment methods such as speckle-interferometry. As expected, the artifact disappears when cross-cleaning is applied to the photon list. Figures $5 \mathrm{a}$ and $5 \mathrm{~b}$ shows the autocorrelations of a simulated flat-field respectively without and with cross-cleaning.

The star-shaped pattern and the central peak in the autocorrelation of images without cross-cleaning are due to the cross-photons. The loss of quantum efficiency due to cross-cleaning is acceptable up to $\bar{N}=4$ photons per frame, with the null-sum test tolerance set to $\varepsilon=2$.

\section{Towards an operational DELTA camera}

This section introduces the engineering characteristics of a prototype presently under construction. 


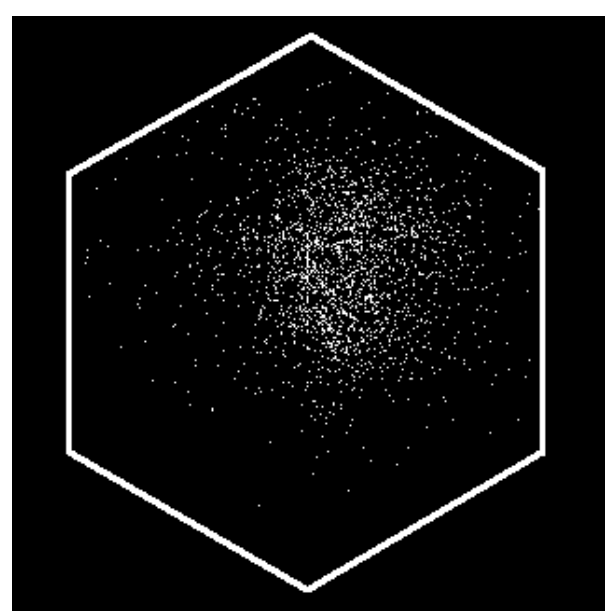

Fig. 4. $10 \mathrm{~ms}$ exposure of an unresolved star in photon counting mode showing the size of the speckle pattern compared with the field of the DELTA camera (the field boundaries are white lines). The original data used for this simulation were acquired with a Ranicon camera

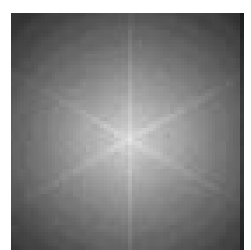

(a)

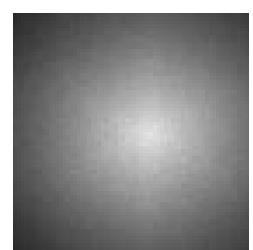

(b)
Fig. 5. Cross-photons generate an artifact in the autocorrelation. This artifact disappears when these are removed form the photon list. Autocorrelations of flat-fields obtained: a) without cross-cleaning, b) with cross-cleaning

\subsection{Choice of a $C C D$}

The characteristics of the linear CCDs used as targets for projections determinate the maximum photon rate, as well as the spatial and temporal resolutions of the camera. The choice of these CCDs is therefore crucial.

The readout frequency of the linear CCDs must be as high as possible to reduce the number of photons per frame at high photon flux and therefore the probability of cross-photons, and to increase the time resolution.

We have chosen the recently released Thomson "Mega Speed" TH7809A. It is a 1024 pixels linear CCD, each pixel being $10 \times 20 \mu \mathrm{m}$ in size, with a $10 \mu \mathrm{m}$ pitch. The TH7809A maximum readout frequency is 400 million pixels per second, thanks to the 16 parallel outputs of an integrated shift register. This shift register allows frame integration during the previous frame readout. As the frame transfer requires less than one clock period, i.e. less than the afterglow of spots caught by the CCDs, no spot will be lost by the frame transfer. The prevention of detecting the same photon in two successive frames is discussed in the next section. The specifications of this CCD chip can be found on the "CCD products" data book from Thomson CSF (1996).

\subsection{Optical setup}

Projections in the DELTA camera are made by three identical optical trains, each one projecting onto a given axis ( $\xi$ in Fig. 6). In the proposed scheme, $\left(L_{1}\right)$ is a spherical collimating lens. $\left(L_{2}\right)$ and $\left(L_{3}\right)$ are cylindric lenses. While $\left(L_{2}\right)$ images in the $\xi$ direction the spot from the front intensifier output onto the linear detector, lens $\left(L_{3}\right)$ images the pupil formed by $\left(L_{2}\right)$. The intensified photon is imaged as a small segment, perpendicular to the CCD line. The image location on the CCD is independent of the spot position along $\eta$ (orthogonal to $\xi$ ), and proportional to the spot position along $\xi$. With dimensions corresponding to commercial grade lenses, the illuminance of one spot onto the CCDs has been evaluated to 4.1 $10^{-2} \mathrm{ph} / \mu \mathrm{m}^{2}=8 \mathrm{ph} /$ pixel (considering a Lambertian emission from the head intensifier output). An auxiliary image intensifier must be placed before each CCD to raise the illuminance over $7.5 \mathrm{ph} / \mu \mathrm{m}^{2}$ (the $\mathrm{TH} 7809 \mathrm{~A}$ readout noise is 300 electrons/pixel, and its quantum efficiency is about $20 \%$ in the wavelengths concerned. Actually, only one auxiliary image intensifier is required if the optical trains are mounted close enough to each other. In this case, the three projections fit in the field of a $25 \mathrm{~mm}$ diameter photocathode.

To match the temporal resolution given by the linear CCDs, the image intensifiers (head and auxiliary) must feature fast decay outputs. The phosphor type P-46 is the most suitable for the auxiliary intensifier, as it provides a 100 ns decay time (from $100 \%$ to $10 \%$ ), and a spectral emission matching the spectral response of the TH7809A better than other fast decay phosphors (P-47 or P-90).

The DELTA camera short frame time and the phosphor afterglow may cause some photo-events occurring at the end of a frame to be still present at the beginning of the next frame. Two solutions can be used to prevent that. The first one is a dead time between frame integrations. This dead time span is a trade-off between the maximum tolerated quantity of photo-events covering two frames, and the minimum desired quantum efficiency. The second solution is to eliminate from a frame the photons having the same coordinates as one in the previous frame. As the probability of having two photons within the same pixel in two consecutive frames is very low (evaluated to $8.810^{-5}$ for $\bar{N}=4$ ), no significant artifact such as those affecting the CP40 would be generated. The same remark can be made for the probability of having two photons within the same pixel in a single frame, evaluated to less than $3.610^{-5}$. 


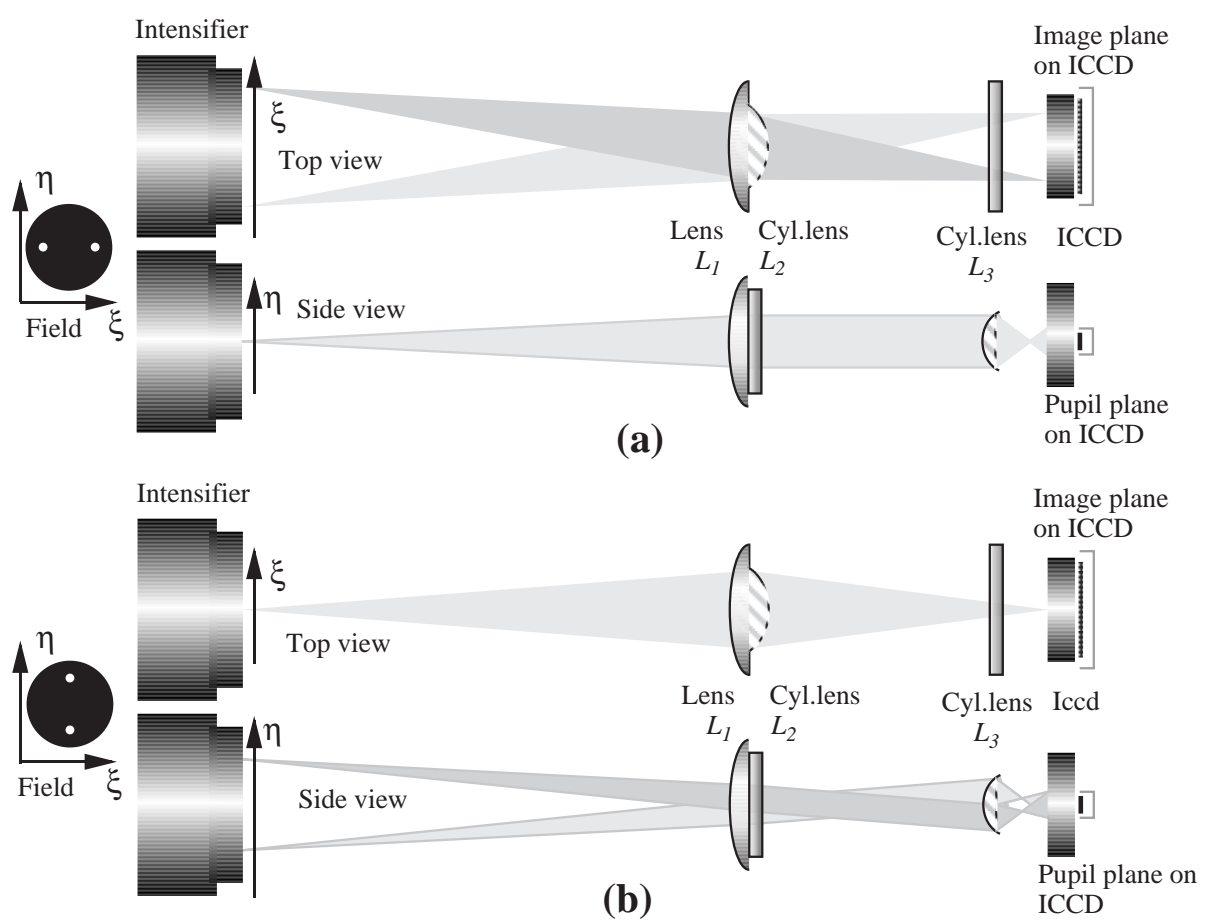

Fig. 6. Scheme of one (among three) optical trains projecting the field onto the CCDs (the other two optical trains are rotated by $\pm 120^{\circ}$ with regard to this one): a) case of two spots separated horizontally in the field (the beam impacts on the CCD are separated), b) case of two spots separated vertically in the field (their impacts on this CCD are superposed)

\subsection{Photon image analysis}

Once projection lists are established for a frame, the next step is the null-sum test. Considering that lists $L_{\mathrm{A}}, L_{\mathrm{B}}$, $L_{\mathrm{C}}$ have the same number $N$ of elements. One could think that scanning all the possible triplets requires a time proportional to $N^{3}$. In fact, this process takes a time proportional to $N^{2}$ : for one of the axes (for example $C$ ), a binarized image of the corresponding CCD line is stored. For each couple $(a, b)$ of elements in $L_{\mathrm{A}}$ and $L_{\mathrm{B}}$, the address $a+b$ in the buffer is probed. If it contains a 1 , photon coordinates $(x=a, y=1.155(a / 2+b))$ are generated. Figure 7 describes the whole process, from CCD acquisition to spatiotemporal photon coordinates. FIFOs and buffer swapping allow a pipe-lined data flow.

With such a design, the prototype should have a $2.6 \mu \mathrm{s}$ temporal resolution, allowing photon flux up to 1.5 million per second with a good quantum efficiency (85\% of the quantum efficiency of a Gen I intensifier) at an average of $\bar{N}=4$ photons per frame. A micro-computer (300 Mips or more) is the simplest way for converting directly digitized CCD signals into photon coordinates.

\subsection{Tuning the camera}

Wrong projection directions and/or displacements of the linear CCDs will cause non-hexagonal field and distorted images. To test the tuning procedures and optical alignment requirements, the software simulator takes into account these parameters. Figure $8 \mathrm{a}$ shows the effect of a misdirected projection: one axis was rotated by only $1^{\circ}$. A similar phenomenon (Fig. 8b) is produced when the three axes have correct angles, but with an angular difference between one axis and its corresponding detector.

Increasing $\varepsilon$ to values higher than 2 is not recommended, since it reduces the resolution, may cause an important loss of quantum efficiency and does not correct image distortion. Using the simulator, we found a simple method for tuning the optical setup, analyzing photon coordinates from a flat-field. This tuning method does not require specific test charts, and can be operated when the camera is docked to a telescope or an interferometer.

\section{Conclusion}

We have proposed a new design for a photon counting device, which can be built from commercially available components. Recent improvements in CCD technology enable the use linear arrays with very fast readout, potentially giving the DELTA camera performance comparable with the best existing photon counting cameras. Moreover, since the DELTA camera provides series of very short frames, it should correctly process quasisimultaneous photons, which may not be detected with asynchronous cameras. Although our DELTA camera is synchronous, it has the advantages of asynchronous cameras: time resolution and high-flux coordinate determination. On-line data processing from the high-rate signals considered here can be done at present by computers, usually tending to be cheaper and faster than custom electronics. This opens a way to "smart detectors", like the DELTA camera, based not only on an electro-optical system, but also on a significant software implementation (as back-projection and cross-cleaning). The DELTA camera 


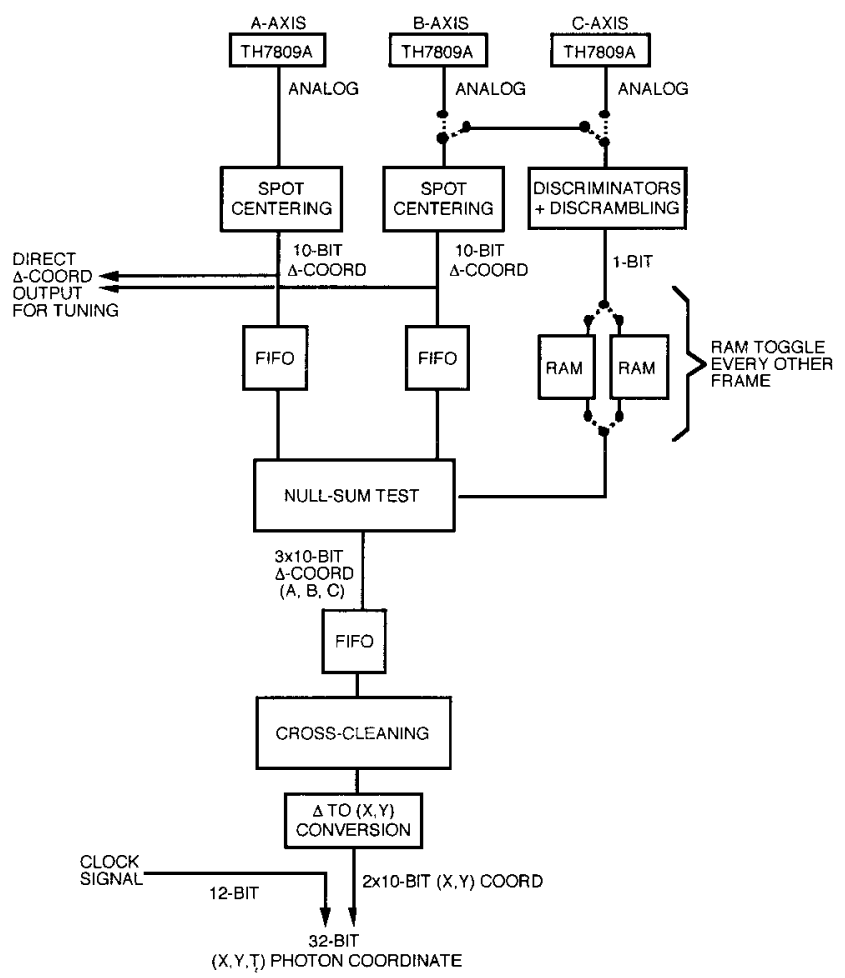

Fig. 7. Flow chart of the process yielding Cartesian $(x, y, t)$ photon coordinates from the linear CCDs. Dashed segments indicate the possible states for switches

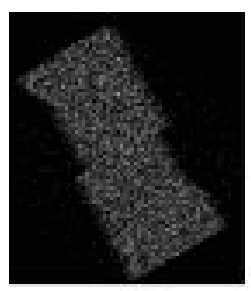

(a)

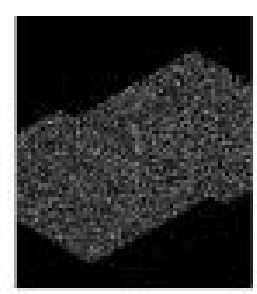

(b)
Fig. 8. Effect of optical misalignments. Flat field images with: a) $1^{\circ}$ misdirected projection, b) $10^{\circ}$ rotation between one axis and its corresponding CCD. The null test tolerance $\varepsilon$ is 4 pixels in these tests. In both cases the size of the field is reduced

prototype under development in our laboratory will, we hope, provide valuable improvements to dark speckle and long baseline interferometry observations in the near future.

Acknowledgements. The authors thank A. Blazit, A. Labeyrie, and F. Vakili, who participated many years ago to the brainstorming which contributed to the birth of the DELTA concept. We are grateful to Dr. J.W. Beletic, and to Dr. O. Jagutzki for their comments and suggestions. S. Morel is supported in this work by a joint fellowship from Matra Marconi Space and CNRS.

\section{References}

Blazit A., Bonneau D., Koechlin L., Labeyrie A., 1977, ApJ 214, L79

Blazit A., 1986, Proc. SPIE 702, 259

Boccaletti A., Labeyrie A., 1997, A\&A (in preparation)

Boksenberg A., Burgess D., 1972, in: Advances in Electronics and Electron Physics 33B, Marton L. (ed.). Academic Press, London, p. 835

Clampin M., Crocker J., Paresce F., Rafal M., 1988, Rev. Sci. Instrum. 59, 1269

Friedman P.G., Cuza R.A., Fleischman, J.R., et al., 1996, Rev. Sci. Instrum. 67, 596

Gezari D.Y., Labeyrie A., Stachnik R., 1972, ApJ 173, L1

Hardy J.W., 1978, Proc. IEEE 66, 651

Knox K.T., Thompson B.J., 1974, ApJ 193, L45

Koechlin L., 1985, J. Opt. (Paris) 16, 269

Koechlin L., Lawson P.R., Mourard D., et al., 1996, Appl. Opt. 35,3002

Labeyrie A., 1970, A\&A 6, 85

Labeyrie A., 1995, A\&A 298, 544

Lampton M., Siegmund O., Raffanti R., 1987, Rev. Sci. Instrum. 58, 2298

Lampton M., Raffanti R., 1994, Rev. Sci. Instrum. 65, 3577

Lawson P.R., 1994, Appl. Opt. 33, 1146

Lohmann A.W., Weigelt G., Wirnitzer B., 1983, Appl. Opt. 22,4028

Martin C., Jelinsky P., Lampton M., Malina R.F., Anger H.O., 1981, Rev. Sci. Instrum. 52, 1067

Morel S., Koechlin L., 1997, Exp. Astron. 7, 117

Mourard D., Tallon-Bosc I., Blazit A., et al., 1994, A\&A 283, 705

Papaliolios C., Mertz L., 1982, Proc. SPIE 331, 360

Raffanti R., Lampton M., 1993, Rev. Sci. Instrum. 64, 1506

Roggemann M.C., Welsh B.M., Fugate R.Q., 1997, Rev. Mod. Phys. 69, 437

Roddier F., 1988, Appl. Opt. 27, 1223

Timothy J.G., Bybee R.L., 1975, Rev. Sci. Instrum. 46, 1615

Timothy J.G., 1983, PASP 95, 810

Timothy J.G., 1985, Opt. Engin. 24, 1066

Vakili F., Koechlin L., 1989, Proc. SPIE 1130, 109 